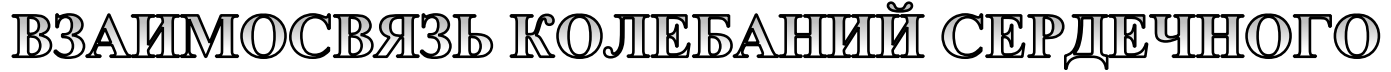

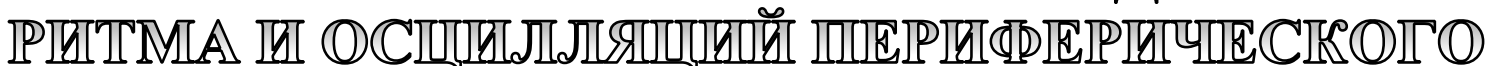

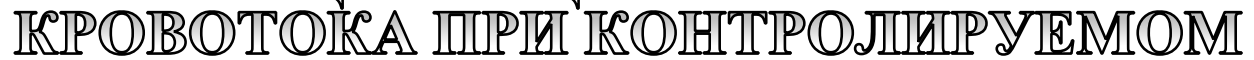

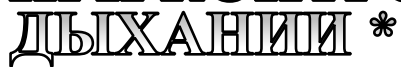

И.А. Мизева, Институт механики сплошных сред УрО РАН

А.В. Танканаг, Институт биофизики клетки РАН

Г.В. Красников, Тульский государственный педагогический университет им. Л.Н. Толстого

Для цитирования:

Мизева И.А., Танканаг А.В., Красников Г.В. Взаимосвязь колебаний сердечного ритма и осцилляций периферического кровотока при контролируемом дыхании // Вестник Пермского федерального исследовательского центра. - 2021. - № 3. - С. 17-22. https://doi.org/10.7242/2658-705X/2021.3.3

Сердечно-сосудистая система человека характеризуется сложным многоуровневым комплексом регуляторных структур с большим количеством внутренних и внешних связей. В фрунциональном аспекте наиболее тесное взаимодействие осуществляется между сердечно-сосудистой и респираторной системами, где важную роль играют нейрогенные регуляторные механизмы, обусловленные активностью симпатического и парасимпатического отделов вегетативной нервной системы. Целью данной работы является изучение характеристик ритмических процессов и их взаимосвязей на центральном и периферическом уровнях сердечно-сосудистой системы. Анализировали вариабельность сердечного ритма (по данным электрокардиографрии), колебания кожной микроциркуляции (по данным фротоплетизмографиии и лазерной допплеровской флоуметрии). Для обработки сигналов использован вейвлет-анализ.

Ключевые слова: сердечно-сосудистая система, контролируемое дыхание, вариации частоты сердечных сокращений, симпатическая нервная система.

\section{Введение}

Для сердечно-сосудистой системы человека характерно наличие ритмических процессов различной природы, изучение которых является целью проекта «Колебания в системе микроциркуляции крови человека в норме и при патологии». Этот проект направлен на экспериментальное исследование колебательных процессов в кровеносной системе как у здоровых добровольцев, так и у пациентов с сахарным диабетом [1], ревматическими заболеваниями [2], псориазом [3] и др. В рамках работы проведено построение

* Работа выполнена при финансовой поддержке РФФИ № 17-41-590560 p-a и № 18-015-00292), авторы благодарят добровольцев, участвовавших в исследовании. 
методов диагностики заболеваний и контроля лечения на основе анализа колебаний в сердечно-сосудистой системе.

Вопрос взаимодействия ритмов дыхания и сердечно-сосудистых колебаний неоднократно рассматривался, начиная с работы [4] изучались вопросы направленности связи [5] и изменения параметров синхронизации с возрастом [6]. В работе [7] изучена роль режима дыхания (спонтанное, контролируемое по частоте и глубине) на параметры синхронизации физиологических ритмов. В серии работ [8-10] развиты методы и подходы для изучения синхронизации и фазового захвата в физиологических сигналах. Особенностью данной работы является детальное изучение синхронизации дыхания, ритма сердца и сигналов периферического кровотока, характеризующих колебания объема и скорости в дистальных сосудах.

Частота сердечных сокращений отражает интегративное влияние различных регуляторных механизмов сердечно-сосудистой системы, что приводит в вариабельности сердечного ритма (ВСР). С одной стороны, дыхание является одним из важнейших факторов, влияющих на ВСР, с другой - тонус периферических сосудов регулируется набором локальных и гуморальных физиологических факторов, которые также могут навязывать колебания физических параметров, описывающих сердечно-сосудистую систему, что проявляется, в том числе, в ВСР.

Дыхание сопровождается респираторной модуляцией центрального артериального и венозного давления, что обусловлено действием дыхательного насоса одного из механизмов венозного возврата крови от периферии к сердцу $[11,12]$. Влияние дыхания на кровоток в микрососудистой системе выражается в амплитудной модуляции пульсовой волны посредством пассивной передачи респираторных модуляций центрального кровяного давления. Кроме того, пульсовая волна может модулироваться респираторно-зависимой активностью симпатической нервной системы через вазомотор- ные механизмы контроля тонуса сосудов, что актуально в случае глубокого медленного дыхания [13]. Ранее было показано, что контролируемое дыхание с частотой ниже частоты спонтанного дыхания приводит к увеличению амплитуды колебаний ВСР, артериального и венозного давления и усиливает симпатическую активность. Отмечается, что амплитуда респираторно-зависимых колебаний ВСР и периферического кровотока увеличивается при глубоком контролируемом дыхании и эффект частотно-зависим [14].

\section{Методика исследования}

В исследовании приняли участие 13 здоровых нормотензивных некурящих добровольцев (3 мужчины и 10 женщин) в

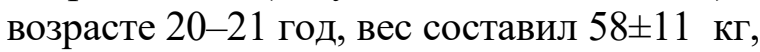
рост $168 \pm 4 \mathrm{~cm}$, частота сердечных сокращений $72 \pm 9$ ударов в минуту). У участников исследования не было выявлено патологий сердечно-сосудистой системы, дыхательных путей и кожных покровов. Из рациона добровольцев были исключены алкоголь, кофеин, вазоактивные медикаменты как минимум за 4 часа до проведения исследования. Измерения проводились при контролируемой температуре $20-24^{\circ} \mathrm{C}$ после адаптации человека к условиям лаборатории. Во время исследования для каждого участника последовательно регистрировали 4 серии пятиминутных записей: первая при спонтанном дыхании, три последующих - при дыхании, контролируемом по глубине и частоте.

В сериях с контролируемым дыханием глубина была фиксированной и составляла $40 \%$ от максимальной жизненной емкости легких, измеряемой методом спирометрии, а частота дыхания составляла 0,04, 0,1 и 0,25 Гц. Для контроля дыхания на грудь испытуемого закреплялся датчик, регистрирующий экскурсии грудной клетки. Кривая с датчика через АЦП выводилась на монитор, и участник исследования старался дышать так, чтобы кривая с грудного датчика воспроизводила референтную кривую, представляющую собой гармонический 
сигнал с заданной частотой. Отметим, что частота спонтанного дыхания обычно составляет $\sim 0,25$ Гц. Во время каждой фазы эксперимента регистрировались сигналы движения грудной клетки (пневмограмма), электрокардиограмма (ЭКГ), фотоплетизмограмма (ФПГ) и колебания объемной скорости кожного кровотока при помощи метода лазерной допплеровской флоуметрии (ЛДФ).

Для определения ВСР зарегистрированные ЭКГ подвергали математической обработке в программе «Поли-Спектр» («Нейрософт», Россия) с целью обнаружения QRS-комплексов и преобразования в последовательности RR-интервалов - кардиоинтервалограммы (КИГ). На рис. 1 фрагменты всех зарегистрированных сигналов для одного участника исследования.

ЛДФ и ФПГ являются стандартными технологиями для исследования состояния микроциркуляторного кровотока. Экспериментальные исследования демонстрируют хорошую корреляцию между этими сигналами для определенных физиологических частотных диапазонов. Несмотря на сходную биофизическую основу этих технологий, различие в используемых физических принципах регистрации оптического сигнала ведет к тому, что соответствующая информация о перфузии получается из разных глубин, что, в свою очередь, приводит к тому, что характеристики сигналов кровотока, зарегистрированных ЛДФ и ФПГ, могут различаться, в частности во время приспособительных (гомеостатических) процессов. Анализ этих различий, на наш взгляд, может предоставить дополнительную информацию о механизмах формирования и взаимосвязи осцилляций кровотока на уровне микроциркуляции.

Для анализа колебаний полученных сигналов применяется вейвлет-анализ, в качестве анализирующей функции выбран вейвлет Морле. Методика подробно описана в работе [1], для количественного анализа изменения энергии пульсаций
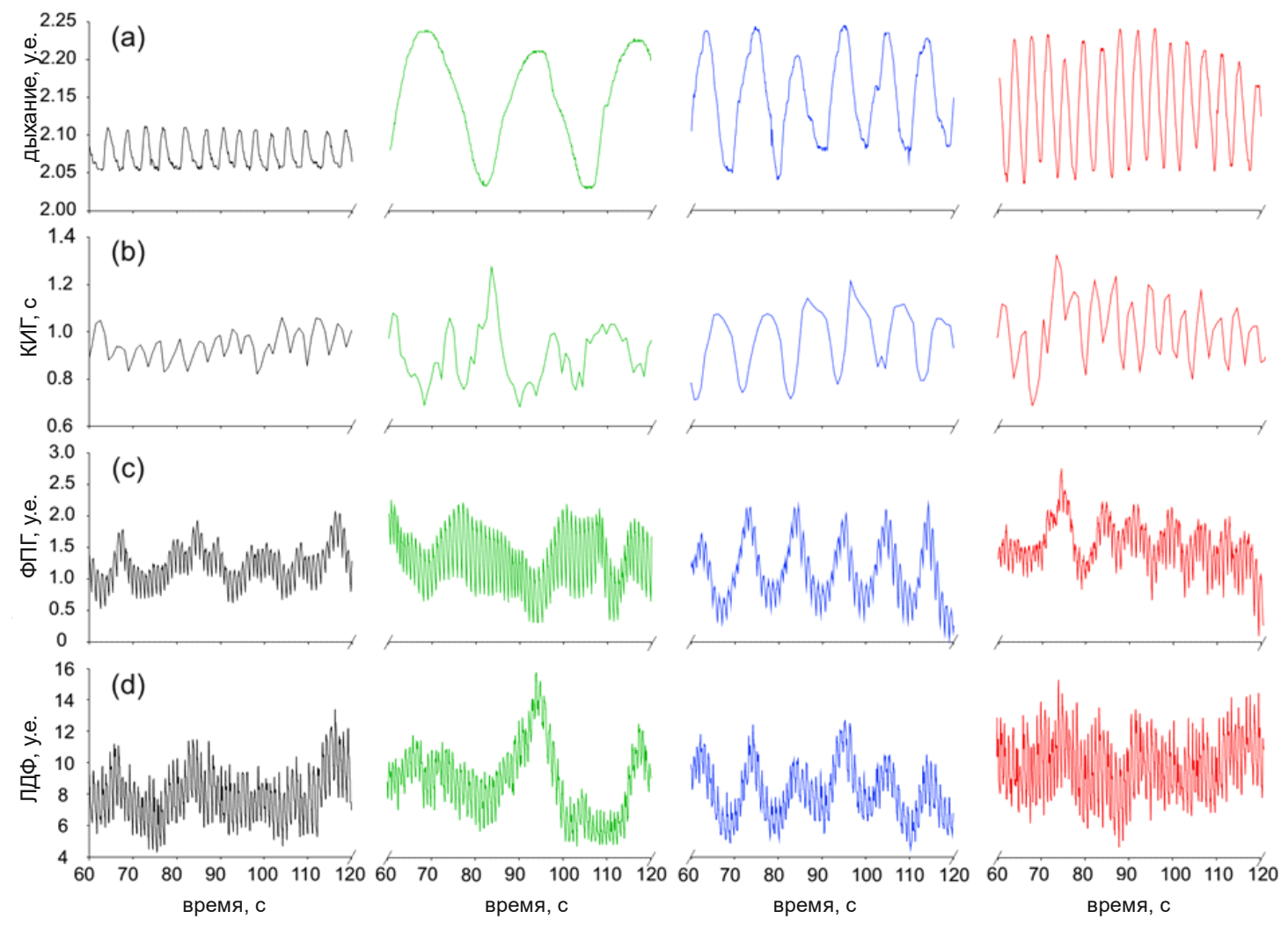

Рис. 1. Характерный вид сигналов дыхания (a), КИГ (b), ЛДФ (c) и ФПГ (d).

Разными ияветами обозначены различные фазы эксперимента: черный - спонтанное дыхание, зеленый-дыхание с частотой 0,04 Ги, синий-0,1 Ги, красный-0,25 Ги 
рассчитывается коэффициент усиления как отношение энергии пульсаций в сигналах КИГ, ЛДФ и ФПГ на частоте контролируемого дыхания к аналогичным параметрам во время спонтанного дыхания.

\section{Результаты}

На рис. 2 представлен коэффициент усиления энергии колебаний в сигналах КИГ, ФПГ и ЛДФ при различных частотах контролируемого дыхания. Анализируя представленные диаграммы, можно отметить, что контролируемое дыхание сопровождается увеличением энергии респираторно-связанных колебаний во всех исследуемых сигналах. При этом в сигналах КИГ и ФПГ (рис. 2, $a, b$ ) эффект имеет частотно-зависимый характер: дыхание с частотой 0,04 и 0,1 Гц вызывает достоверно большее увеличение энергии пульсации на соответствующей частоте дыхания по сравнению с частотой дыхания 0,25 Гц. В сигнале ЛДФ, напротив, увеличение энергии колебаний не зависит от частоты дыхания (рис. 2, c). Дыхание с часто- той 0,04 и 0,1 Гц вызывает значительное увеличение энергии пульсации в сигналах КИГ и ФПГ (рис. $2 a, b$ ) на данных частотах, причем этот коэффициент значимо выше, чем для частоты 0,25 Гц. В сигнале ЛДФ увеличение энергии колебаний не зависит от частоты дыхания (рис. 2,c).

Для оценки взаимосвязи колебаний на различных частотах был использован вейвлет кросс-корреляционный анализ [15]. На рис. 3 и 4 показаны осредненные по группе вейвлет кросс-корреляции пар анализируемых сигналов при спонтанном и контролируемом дыхании. Для всех режимов контролируемого дыхания наблюдается значимое увеличение корреляции колебаний в парах дыхание - КИГ, дыхание-ФПГ и дыхание - ЛДФ на частоте дыхания (рис. 3). Обнаружено, что при дыхании с частотой 0,4 и 0,1 Гц корреляции в парах КИГ - ФПГ и КИГ - ЛДФ значимо нарастают (рис. 4). В паре ЛДФ ФПГ корреляции достаточно высокие и при спонтанном дыхании, и контролируемое дыхание не приводит к значимому увеличению корреляции (рис. 4).
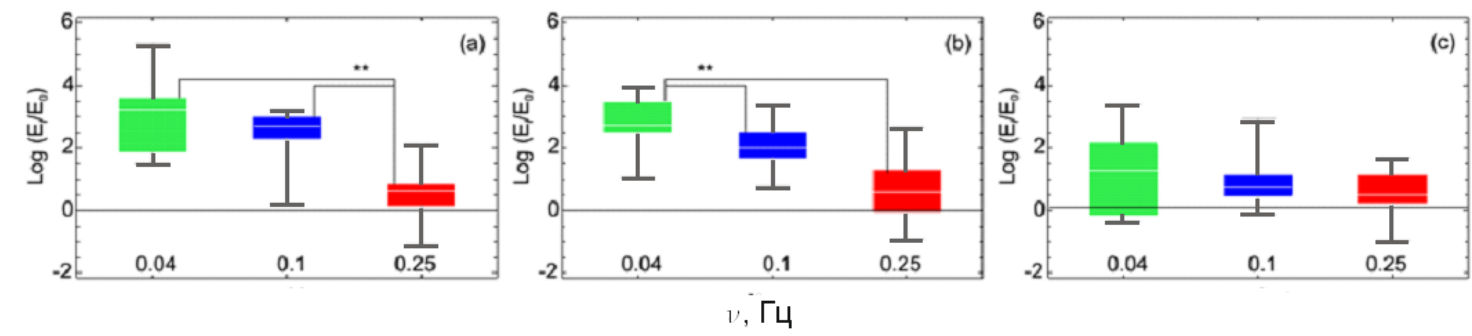

Рис. 2. Диаграмма размаха для изменения энергии колебаний КИГ и периферического кровотока при разных частотах контролируемого дыхания по сравнению со спонтанным дыханием. КИГ (a), ЛДФ (b), ФПГ (c). Разными цвветами обозначены различные фазы эксперимента: зеленый-dыхание с частотой 0,04 Ги, синий - 0,1 Ги, красный-0,25 Ги.

Здесь и далее на диаграммах размаха границы прямоугольников показаны 25\%, 50\%, 75\%, усы соответствуют наблюдаемым минимумам и максимумам распределения. Символ ** соответствует $p<0,01$
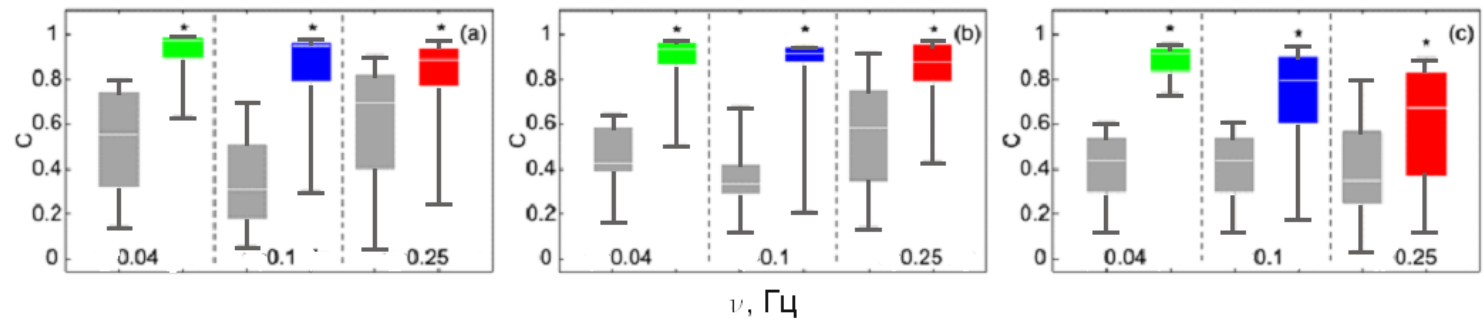

Рис. 3. Вейвлет-корреляция сигналов дыхания с КИГ (a), ЛДФ (b), ФПГ (c) при спонтанном (серый) и контролируемом (зеленый-0,04 Ги, синий-0,1 Ги, красный-0,25 Ги) дыхании.

Символ * здесь и далее обозначает $p<0,05$ 

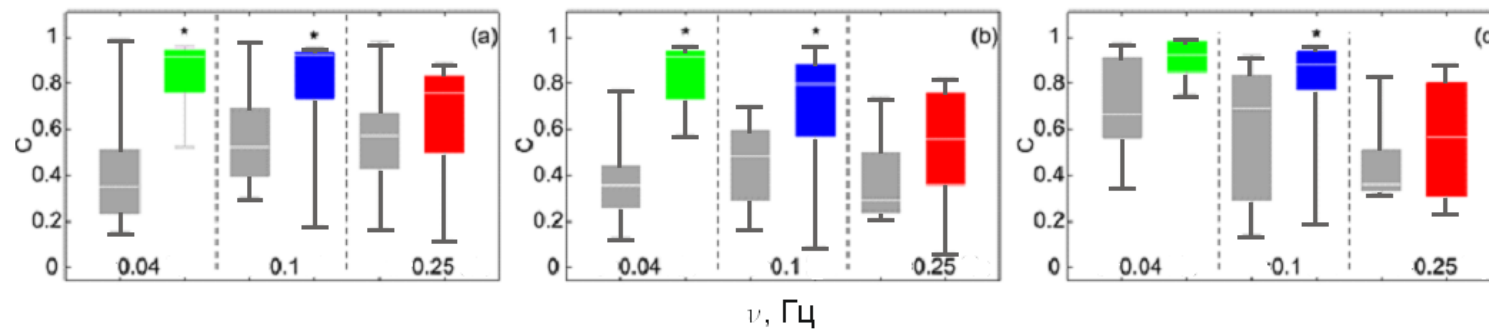

Рис. 4. Вейвлет-корреляциия следующих пар сигналов: КИГ-ФПГ (а), КИГ-ЛДФ (b), ФПГ-ЛДФ (c)

\section{Обсуждение и выводы}

В рамках работы был создан уникальный экспериментальный протокол дыхательного теста, который позволил контролировать как частоту, так и глубину дыхания, опираясь на данные грудного датчика, показания которого выводились на монитор компьютера и сравнивались с опорным сигналом в режиме реального времени. Глубина дыхания во всех тестах была одинаковой, а изменялась только частота.

Установлено, что колебания во всех трех зарегистрированных сигналах коррелируют с дыханием, кроме того, существует высокая корреляция в парах КИГ -
ФПГ, КИГ - ЛДФ и ФПГ - ЛДФ при контролируемом дыхании с 0,04 и 0,1 Гц. Эта корреляция значительно выше, чем при спонтанном дыхании. При контролируемом дыхании с частотой 0,25 Гц, близкой к частоте спонтанного дыхания, мы не обнаружили существенного увеличения корреляции в исследуемых парах сигналов на заданных частотах и предполагаем, что выявленные особенности обусловлены в первую очередь физиологическими особенностями формирования сигналов ФПГ и ЛДФ, в частности влиянием симпатической нервной системы на тонус мелких сосудов.

\section{Библиографический список}

1. Mizeva I., Zharkikh E., Dremin V., Zherebtsov E., Makovik I., Potapova E., Dunaev A. Spectral analysis of the blood flow in the foot microvascular bed during thermal testing in patients with diabetes mellitus // Microvascular research. - 2018. - Vol. 120. - P. 13-20.

2. Mizeva I., Makovik I., Dunaev A., Krupatkin A., Meglinski I. Analysis of skin blood microflow oscillations in patients with rheumatic diseases // Journal of Biomedical Optics. - 2017. - Vol. 22(7). - 070501.

3. Потапова Е.В., Филина М.А., Козлов И.О., Жарких Е.В., Дрёмин В.В., Малая Н.С., Снимщикова И.А., Дунаев А.В., Сидоров В.В., Крупаткин А.И. Особенности локальной микроциркуляции крови у пациентов с псориазом // Регионарное кровообращение и микроциркуляция. - 2018. T. 17. - № 3. - C. 58-64.

4. Schäfer C., Rosenblum M.G., Abel H.-H., Kurths J. Synchronization in the Human Cardiorespiratory System // Physical Review E. - 1999. -Vol. 60. - P. 857-870.

5. Rosenblum M.G., Cimponeriu L., Bezerianos A., Patzak A., Mrowka R. Identification of coupling direction: Application to cardiorespiratory interaction// Physical Review E. - 2002. - Vol. 65. - P. 041909.

6. Shiogai Y., Stefanovska A., McClintock P.V.E. Nonlinear dynamics of cardiovascular ageing // Physics Reports. - 2010. - Vol .488. - P. 51-110.

7. Prokhorov M.D., Ponomarenko V.I., Gridnev V.I., Bodrov M.B., Bespyatov A.B. Synchronization between main rhythmic processes in the human cardiovascular system // Phys. Rev. E. - 2003. - Vol. 68. - 041913.

8. Боровкова Е.И., Караваев А.С., Пономаренко В.И., Прохоров М.Д, Безручко Б.П. Диагностика частотного захвата в условиях воздействия сигналом переменной частоты // Изв. РАН. Сер. Физическая. - 2011. - Т. 75. - № 12. - С. 1704-1708.

9. Караваев А.С., Киселев А.Р., Гриднев В.И., Боровкова Е.И., Прохоров М.Д., Посненкова О.М., Пономаренко В.И., Безручко Б.П., Швари В.А. Фазовый и частотный захват 0,1 Гц колебаний в ритме сердца и барорефлекторной регуляции артериального давления дыханием с линейно меняющейся частотой у здоровых лиц // Физиология человека. - 2013. - Т. 39. - № 4. - С. 105-111.

10. Karavaev A.S., Kiselev A.R., Runnova A.E., Zhuravlev M.O., Borovkova E.I., Prokhorov M.D., Ponomarenko V.I., Pchelintseva S.V., Efremova T.Yu., Koronovskii A.A., Hramov A.E. Synchronization of infra-slow oscillations of brain potentials with respiration // Chaos. - 2018. - Vol. 28. - P. 081102. 
11. Meredith D.J., Clifton D., Charlton P., Brooks J., Pugh C.W., Tarassenko L. Photoplethysmographic derivation of respiratory rate: a review of relevant physiology // Journal of Medical Engineering \& Technology. - 2012. - Vol. 36 (1).

12. Bollinger A., Yanar A., Hoffmann U., Franzeck $U$. K. Is high-frequency flux motion due to respiration or to vasomotion activity? in: C. Allegra, M. Intaglietta, K. Messmer (eds.), Vasomotion and Flow Motion. 1993. - Vol. 20. - P. 52-58.

13. Russo M.A., Santarelli D.M., ORourke D. The physiological effects of slow breathing in the healthy human // Breathe. - 2017. - Vol. 13 (4). - P. 298-309.

14. Krasnikov G.V., Tyurina M.Y., Tankanag A.V., Piskunova G.M., Chemeris N.K. Analysis of heart rate variability and skin blood flow oscillations under deep controlled breathing. // Respiratory Physiology and Neurobiology. - 2013. - Vol. 185 (3). - P. 562-570.

15. Tankanag A., Krasnikov G., Mizeva I. A pilot study: Wavelet cross-correlation of cardiovascular oscillations under controlled respiration in humans // Microvascular research. - 2020. - Vol. 130. - 103993.

\title{
RELATION OF CARDIOVASCULAR OSCILLATIONS UNDER CONTROLLED RESPIRATION IN HUMANS
}

\author{
I.A. Mizeva ${ }^{1}$, A.V. Tankanag ${ }^{2}$, G.V. Krasnikov ${ }^{3}$ \\ ${ }^{1}$ Institute of Continuous Media Mechanics UB RAS \\ ${ }^{2}$ Institute of Cell Biophysics RAS, Pushchino \\ ${ }^{3}$ Tula State Lev Tolstoy Pedagogical University
}

\section{For citation:}

Mizeva I.A., Tankanag A.V., Krasnikov G.V. Relation of cardiovascular oscillations under controlled respiration in humans /I Perm Federal Research Center Journal. - 2021 . № 3. - P. 17-22. https://doi.org/10.7242/2658-705X/2021.3.3

The human cardiovascular system is characterized by multi-level complex of regulatory structures with a large number of internal and external connections. In the functional aspect, the closest interaction is carried out between the cardiovascular and respiratory systems, where neurogenic regulatory mechanisms play an important role due to the activity of the sympathetic and parasympathetic parts of the autonomic nervous system. The aim of this work is to study the characteristics of rhythmic processes and their relationships in various parts of the cardiovascular system recorded using non-invasive methods (ECG, photoplethysmography and laser Doppler flowmetry). For signal processing, wavelet analysis is used.

Keywords: cardiovascular system, controlled respiration, heart rate variability, autonomic nervous system.

\section{Сведения об авторах}

Мизева Ирина Андреевна, кандидат физико-математических наук, научный сотрудник лаборатории физической гидродинамики, Институт механики сплошных сред УрО РАН (ИМСС УрО РАН), 614013, г. Пермь, ул. Академика Королева, 1; e-mail: mizeva@icmm.ru

Танканаг Арина Владимировна, кандидат биологических наук, ведущий научный сотрудник лаборатории клеточной нейробиологии, Институт биофизики клетки РАН - обособленное подразделение Пущинского научного центра биологических исследований РАН (ИБК РАН), 142290, г. Пущино Московской области, Институтская, 3; e-mail: tav@icb.psn.ru

Геннадий Викторович Красников, кандидат биологических наук, доцент кафедры медикобиологических дисциплин и фармакогнозии, Тульский государственный педагогический университет им. Л.Н. Толстого (ТГПУ им. Л.Н. Толстого), 300026, Тульская обл., г. Тула, просп. Ленина, д. 125.; e-mail: gvkrasnikov@gmail.com 